\title{
Effects of Noninvasive Versus Invasive Mechanical Ventilation on Sleep in the Intensive Care Unit - A Pilot Study
}

\author{
Authors:

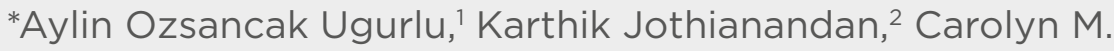 \\ D'Ambrosio, ${ }^{3}$ Samy Sidhom, ${ }^{4}$ Eric Garpestad, ${ }^{5}$ Nicholas S. Hill ${ }^{5}$ \\ 1. Başkent University Department of Pulmonary Disease, Istanbul, Turkey \\ 2. Maurey Regional Medical Center, Columbia, Tennessee, USA \\ 3. Brigham and Women's Hospital, Boston, Massachusetts, USA \\ 4. Atrius Health, Boston, Massachusetts, USA \\ 5. Tufts Medical Center Department of Pulmonary, Critical Care and Sleep Medicine, \\ Boston, Massachusetts, USA \\ *Correspondence to aozsancak@hotmail.com
}

Disclosure: $\quad$ Prof Hill has been a consultant for and has received honoraria for talks from Philips Respironics. The other authors have declared no conflicts of interest.

Acknowledgement: This study is registered in clinicaltrials.gov (NCTO0638339).

Received:

14.01.20

Accepted:

25.03.20

Keywords:

Intensive care unit (ICU), invasive mechanical ventilation (INV), noninvasive ventilation (NIV), polysomnography (PSG).

Citation:

EMJ Respir. 2020;8[1]:127-136.

\section{Abstract}

Rationale: Use of noninvasive ventilation (NIV) has increased in intensive care units, but sleep during NIV has received little attention. The authors surmised that due to frequent air leaks and mask discomfort, patients receiving NIV would manifest poorer sleep quality than those receiving invasive mechanical ventilation (INV).

Methods: A prospective observational study on patients receiving NIV or INV for respiratory failure in a medical intensive care unit or coronary care unit. Patients were monitored by polysomnography for 24 hours with simultaneous collection of data on ventilator and environmental parameters.

Results: Eight subjects in each group were studied. Mean total sleep time was $7.29+1.78$ hours (range: 0.57-13.82) in the NIV versus $11.74+0.65$ hours (8.95-15.19) in the INV group $(p=0.034)$. Sleep efficiency was lower in NIV than INV group (30.4\% versus $53.3 \%$, respectively; $p=0.013$ ). The NIV group had lighter sleep than the INV group (mean \% of Stage 1: 36.9\% versus $17.2 \%$ of total sleep time, respectively; $\mathrm{p}=0.000$ ), whereas no significant differences were found for other stages. Median total arousal and awakening indexes were higher in the NIV group (16.8/hour versus 4.4/hour and 5.3/hour versus $2.1 /$ hour, respectively; $p=0.005)$, as well as spontaneous arousals and awakenings $(p=0.006$ and $p=0.005$, respectively). Sedation was provided mostly by intermittent bolus in the NIV group whereas often by infusion in the INV group.

Conclusion: Compared to INV, NIV in critically ill patients was associated with poorer quality and quantity of sleep. Future studies should determine whether adjustments in ventilator settings, mask type or fit, or use of sedation/analgaesia can improve sleep in patients receiving NIV. 


\section{INTRODUCTION}

Disruption of sleep is remarkably common in critically ill patients. ${ }^{1-3}$ Abnormalities of sleep in this population include sleep deprivation, sleep fragmentation, and alterations in sleep architecture and circadian rhythm. These abnormalities may lead to undesirable consequences, such as delirium, delayed weaning in intensive care units (ICU), or late noninvasive ventilation (NIV) failures. ${ }^{4-6}$ Mechanical ventilation is one of the important factors associated with sleep disturbances in the ICU., 3,7,

NIV has been increasingly used in ICU. ${ }^{9-11}$ Although recognition of the importance of poor sleep in mechanical ventilation patients is increasing, only a few studies have investigated the effect of NIV on sleep in patients with acute respiratory failure (ARF). 5,12 Earlier studies focussed on patients with chronic respiratory failure. ${ }^{13,14}$ Air leaking was associated with frequent arousals during lighter stages of sleep and decreased sleep efficiency and durations of slow-wave sleep (SWS) and rapid eye movement (REM) sleep. ${ }^{13,14}$ Sleep studies on hypercapnic ARF patients receiving NIV in the ICU showed similar sleep architecture changes., ${ }^{5,12}$

The authors hypothesised that because of frequent air leaks and mask discomfort, patients receiving NIV would manifest poorer sleep quality than those receiving invasive mechanical ventilation (INV). Therefore, they aimed to define and compare sleep characteristics in critically ill patients receiving NIV and INV during a 24-hour monitoring period.

\section{METHODS}

\section{Patients}

This prospective, observational, pilot study was conducted in critically ill adult patients undergoing INV or NIV for ARF in the medical ICU unit or coronary care unit (CCU) at Tufts Medical Center, Boston, Massachusetts, USA. The Institutional Review Board of Tufts Medical Center approved the study (Tufts ID \#8053). ${ }^{15}$ Written informed consent was obtained from all participants or their families.

All adult patients receiving NIV or INV for ARF in the medical ICU or CCU and anticipated to be continuing NIV for $>8$ hours/24 hours or INV for 24 hours were eligible and screened on the days when the primary researcher ( $\mathrm{Dr}$ Ugurlu) was available. All patients were in private rooms that could be isolated from the nursing station through a sliding glass door.

Exclusion criteria are listed as presence of premorbid diseases that could interfere with application or interpretation of sleep monitoring (including central nervous system disorders, dementia, known sleep disorders); previous home treatment with bilevel positive airway pressure (BiPAP) or continuous positive airway pressure; depressed sensorium (Riker score: $\leq 2$ ); presence of head trauma, psychiatric illness, anoxic brain injury, drug overdose, or uncontrolled seizure disorder; severe haemodynamic instability; recalcitrant hypoxaemia (inability to sustain oxygen saturation $>88 \%$ ); and tracheostomy.

All patient care decisions (supportive care and mechanical ventilation) were made by the ICU team and not by any investigator during the study. Respiratory therapists, nurses, and/ or critical care physicians periodically checked patients to ensure that the subjects were adapted to the NIV use (proper mask fit, synchrony with ventilation, etc).

\section{Polysomnography and Scoring}

Continuous 24-hour standard polysomnography (PSG) utilising portable noninvasive PSG monitoring equipment (Grass Comet, West Warwick, Rhode Island, USA) was conducted with simultaneous monitoring of noise. The staff attending the study fixed displacing leads.

Sleep studies were continuously attended and scored manually according to standard criteria ${ }^{16,17}$ by a registered PSG technologist and then reviewed in total by a sleep specialist blinded to mode of ventilation (Dr D'Ambrosio). Circadian sleep distribution was evaluated by separating the total recording time into a daytime segment (6 AM-10 PM) and a nocturnal segment (10 PM-6 AM).

Sleep efficiency was defined as the ratio of time asleep to total recording time. Total sleep time (TST) was defined as the sum of total time spent in any sleep stage during total recording time. The percentage of time spent in each sleep stage during TST was calculated. The sleep 
fragmentation index was defined as the number of arousals and awakenings per hour of sleep. Arousals and awakenings caused by noise, patient care activities, patient-ventilator asynchronies (including auto-triggering, ineffective triggering, and double triggering, based on inductance plethysmography and airway pressure measurements), or mask leak (based on leak and airway pressure monitoring) were defined as those occurring within 3 seconds of termination of these events. The rest of the arousals or awakenings were defined as spontaneous.

\section{Environmental Monitoring}

Environmental noise was continuously monitored by a sound level meter (Extech Instruments, Nashua, New Hampshire, USA) secured to the head of the bed, and simultaneously recorded on the PSG. The ceiling fluorescent light turned on throughout the daytime and turned off at night, except during nursing care. The bedside environment was monitored by the staff, who recorded the time and duration of all activities (including interactions between the patients and the visitors or critical care team member, nursing care, and procedures) on the patient's bedside diary.

\section{Data Collection}

Data included demographics, date and time of hospital and ICU/CCU admission, admitting diagnosis, causal diagnosis, and reason for mechanical ventilation. Further data included type of mechanical ventilation, settings, sedation level, medications, last arterial blood gas measurement, and last chest $X$-ray findings. Acute physiology and chronic health evaluation II (APACHE II) score was calculated on the day of the sleep study using the most abnormal values during the 24-hour PSG period. Additionally, the investigator attending the sleep study recorded all pertinent events and times in a bedside log.

\section{Statistical Analysis}

This study was performed as a pilot and no previous study has compared sleep patterns during NIV and INV in critically ill patients. Accordingly, no power analysis was proposed. Statistical analysis was performed using SPSS statistical software (SPSS 17.0, Chicago, Illinois, USA). Continuous variables were expressed as median (interquartile range) or mean \pm standard error of mean with minimum and maximum values, based on the distribution of data. Variables were compared using chi-squared test or MannWhitney $U$ test. A two-tailed $\mathrm{p}$ value $<0.05$ was considered statistically significant.

\section{RESULTS}

\section{Patient Demographics}

Out of 383 patients with ARF anticipated to continue using NIV or INV for $\geq 24$ hours, 346 patients were excluded (central nervous system disorder/injury: 170; depressed sensorium: 51; tracheostomised: 33; previous home continuous positive airway pressure/BiPAP: 26; known sleep disorder: 22 [not using NIV at home]; severe haemodynamic instability: 17; unstable status: 17; legal issues: five [e.g., prisoner, no relatives]; diffuse skin lesions: three; recalcitrant hypoxaemia: two). Furthermore, 17 patients refused enrolment. Overall, 20 patients were enrolled to the study; however, a total of four patients were excluded after enrolment because of early extubation ( $<24$ hours) in the INV group (three patients) or early intubation $(<24 \mathrm{~h})$ in the NIV group (one patient).

The study population characteristics are provided in Table 1. Chronic obstructive pulmonary disease and heart diseases (including coronary arterial disease, rhythm disorders, and congestive heart failure) were the predominant comorbidities in both the NIV and INV groups (Table 1). Although there was no statistically significant difference in the comparison of causal diagnoses, there tended to be more acute-on-chronic respiratory failure in the NIV group and more de novo respiratory failure in the INV group.

\section{Characteristics of Mechanical Ventilation at Initiation of the Study}

All patients in the NIV group were on BiPAP delivered through dedicated ventilators. Mean inspiratory and expiratory positive airway

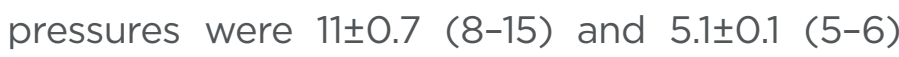
$\mathrm{CmH}_{2} \mathrm{O}$, respectively. All INV patients were on volume-limited assist-control ventilation with a mean tidal volume of 507.9 \pm 24.6 (350-550) mL ( $7.1 \pm 0.3 \mathrm{~mL} / \mathrm{kg}$ per ideal body weight), similar to NIV patients (441.3 \pm 24.4 [316-512] $\mathrm{mL}$ [7.8 \pm 0.7 $\mathrm{mL} / \mathrm{kg}$ per ideal body weight]). 
Table 1: Characteristics of the patients $(\mathrm{N}=16)$.

\begin{tabular}{|c|c|c|c|}
\hline & $\begin{array}{c}\text { Noninvasive ventilation } \\
\text { group } \\
(n=8)\end{array}$ & $\begin{array}{l}\text { Invasive mechanical } \\
\text { ventilation group } \\
\qquad(n=8)\end{array}$ & $p$ value \\
\hline Age (years) & $72.6 \pm 3.1(54-85)$ & $64.1 \pm 4.6(38-78)$ & NS \\
\hline Male $(n[\%])$ & $3(37.5)$ & $8(100)$ & 0.013 \\
\hline BMI $\left(\mathrm{kg} / \mathrm{m}^{2}\right)$ & $25.7 \pm 6.37(16.2-36.0)$ & $26.8 \pm 8.22(16.9-44.3)$ & NS \\
\hline Location (MICU/CCU) & $5 / 3$ & $5 / 3$ & NS \\
\hline Causes of ARF (n) & & & NS \\
\hline Acute pulmonary oedema & 2 & 1 & \\
\hline ARDS & 0 & 2 & \\
\hline Cardiopulmonary arrest & 0 & 1 & \\
\hline COPD exacerbation & 1 & 0 & \\
\hline Haemoptysis & 0 & 1 & \\
\hline Lung cancer & 0 & 1 & \\
\hline Neuromuscular disease & 1 & 0 & \\
\hline Pneumonia & 1 & 1 & \\
\hline Pulmonary emboli & 1 & 0 & \\
\hline Restrictive lung disease* & 2 & 0 & \\
\hline Sepsis & 0 & 1 & \\
\hline $\begin{array}{l}\text { At the initiation of study: } \\
\text { duration of } \mathrm{MV}^{+} \text {(day) }\end{array}$ & $1(1.00-3.25)$ & $4(1.00-5.00)$ & NS \\
\hline Apache II score & $20.4 \pm 2.3(13-28)$ & $13.4 \pm 1.9(6-21)$ & 0.039 \\
\hline Vitals & & & NS \\
\hline Heart rate $(\mathrm{bpm})$ & $83.6 \pm 6.1(58-105)$ & $83.5 \pm 3.5(68-97)$ & \\
\hline Respiratory rate (bpm) & $22.1 \pm 2.4(10-32)$ & $20.1 \pm 1.5(13-25)$ & \\
\hline $\begin{array}{l}\text { Systolic blood pressure } \\
(\mathrm{mmHg})\end{array}$ & $106.5 \pm 5.3(90-129)$ & $114.8 \pm 6.3(90-140)$ & \\
\hline $\begin{array}{l}\text { Diastolic blood pressure } \\
(\mathrm{mmHg})\end{array}$ & $55.6 \pm 5.2(38-81)$ & $56.9 \pm 3.2(42-66)$ & \\
\hline \multicolumn{4}{|l|}{ Arterial blood gases } \\
\hline $\mathrm{pH}$ & $7.25 \pm 0.03(7.26 \pm 7.48)$ & $7.44 \pm 0.01(7.36-7.49)$ & 0.022 \\
\hline $\mathrm{P}_{\mathrm{a}} \mathrm{CO}_{2}$ & $49.4 \pm 5.6(34-68)$ & $42.4 \pm 1.1(38-48)$ & NS \\
\hline $\mathrm{P}_{\mathrm{a}} \mathrm{O}_{2} / \mathrm{FiO}_{2}^{+}$ & $290(220-410)$ & $180(160-240)$ & NS \\
\hline
\end{tabular}

*Restrictive lung disease was due to pleural effusion.

+Values are given in median (IQR). The rest of the values were given as mean \pm SEM (min-max).

ARDS: acute respiratory distress syndrome; ARF: acute respiratory failure; bpm: beats per minute; CCU: coronary care unit; COPD: chronic obstructive pulmonary disease; $\mathrm{FiO}_{2}$ : fraction of inspired oxygen; IQR: interquartile range; max: maximum; MICU: medical intensive care unit; min: minimum; MV: mechanical ventilation; NS: not significant ( $p>0.05$ ); $\mathrm{P}_{\mathrm{a}} \mathrm{CO}_{2}$ : partial pressure of carbon dioxide; $\mathrm{P}_{\mathrm{a}} \mathrm{O}_{2}$ : partial pressure of oxygen; SEM: standard error of the mean.

Mean fraction of inspired oxygen and mean set respiratory rate in the NIV and INV groups were $52.5 \pm 7.5$ versus $40 \pm 3.3 \% \quad(p=0.14)$ and $10.5 \pm 1.1$ versus $13.3 \pm 0.8$ beats per minute $(p=0.06)$, respectively.

\section{Sleep Architecture of Patients}

Compared to INV patients, NIV patients slept less and had lower sleep efficiency (Table 2). 
Table 2: Comparison of sleep characteristics between patients treated with noninvasive ventilation and invasive mechanical ventilation.

\begin{tabular}{|c|c|c|c|}
\hline & $\begin{array}{c}\text { Noninvasive ventilation } \\
\text { group }\end{array}$ & $\begin{array}{l}\text { Invasive mechanical } \\
\text { ventilation group }\end{array}$ & $\mathrm{p}$ value \\
\hline TST (hours) & $7.3 \pm 1.8(0.6-13.8)$ & $11.7 \pm 0.7(9.0-15.2)$ & 0.034 \\
\hline Day/night (\% of TST) & $57 / 43$ & $60 / 40$ & NS \\
\hline \multicolumn{4}{|l|}{ Sleep efficiency (\%) } \\
\hline Total & $30.4 \pm 7.5(2.4-57.6)$ & $53.3 \pm 3.1(37.5-64.0)$ & 0.013 \\
\hline Daytime (6 AM-10 PM) & $23.5 \pm 6.4(3.7-51.6)$ & $50.1 \pm 2.8(34.9-57.4)$ & $<0.001$ \\
\hline Night-time (10 PM-6 AM) & $44.2 \pm 10.3(0.0-74)$ & $58.9 \pm 5.0(36.0-77.3)$ & NS \\
\hline Stage 1 (\% of TST) & $36.9 \pm 3.3(23.7-53.0)$ & $17.2 \pm 2.8(3.3-26.9)$ & $<0.001$ \\
\hline Stage 2 (\% of TST) & $57.1 \pm 3.1(46.2-70.6)$ & $64.8 \pm 6.2(26.4-86.8)$ & NS \\
\hline SWS* (\% of TST) & 1.4 (IQR 0.0-4.5) & 9.5 (IQR 5.5-17.3) & 0.04 \\
\hline $\mathrm{REM}^{*}(\%$ of TST) & 0.6 (IQR 0.0-2.9) & 0.0 (IQR 0.0-1.1) & NS \\
\hline $\begin{array}{l}\text { Total fragmentation } \\
\text { index/hour }\end{array}$ & 22.4 (IQR 14.0-39.5) & 6.3 (IQR 5.2-11.4) & 0.003 \\
\hline Arousal index*/hour & 16.8 (IQR 9.1-31.8) & 4.4 (IQR 3.7-8.5) & 0.005 \\
\hline Spontaneous arousal index* & 14.1 (IQR 7.4-17.9) & 3.2 (IQR 2.2-4.2) & 0.006 \\
\hline $\begin{array}{l}\text { Ventilator asynchrony-air leak } \\
\text { associated arousal index* }\end{array}$ & 2.2 (IQR 0.1-10.9) & 0.1 (IQR 0.0-1.5) & NS \\
\hline $\begin{array}{l}\text { Noise associated arousal } \\
\text { index* }\end{array}$ & 0.7 (IQR 0.1-1.1) & 0.4 (IQR 0.2-0.5) & NS \\
\hline $\begin{array}{l}\text { Patient care associated } \\
\text { arousal index* }\end{array}$ & 0.5 (IQR 0.0-1.2) & 0.6 (IQR 0.3-1.2) & NS \\
\hline Awakening index*/hour & 5.3 (IQR 4.9-6.8) & 2.1 (IQR 1.7-3.0) & 0.005 \\
\hline $\begin{array}{l}\text { Spontaneous awakening } \\
\text { index* }\end{array}$ & 2.6 (IQR 2.0-4.9) & 1.0 (IQR 0.6-1.4) & 0.005 \\
\hline $\begin{array}{l}\text { Ventilator asynchrony-air leak } \\
\text { associated awakening index* }\end{array}$ & 1.4 (IQR 0.0-1.7) & 0.2 (IQR 0.1-0.2) & NS \\
\hline $\begin{array}{l}\text { Noise associated awakening } \\
\text { index* }\end{array}$ & 0.4 (IQR 0.1-0.7) & 0.3 (IQR 0.1-0.3) & NS \\
\hline $\begin{array}{l}\text { Patient care associated } \\
\text { awakening index }\end{array}$ & $0.5 \pm 0.2(0.0-1.7)$ & $0.8 \pm 0.1(0.3-1.4)$ & NS \\
\hline
\end{tabular}

*Values are given in median (IQR). Rest of the values are given as mean \pm SEM (min-max). IQR: interquartile range; max: maximum; min: minimum; NS: not significant ( $p>0.05)$; REM: rapid eye movement; SEM: standard error of the mean; SWS: slow-wave sleep; TST: total sleep time.

Sleep efficiency was significantly lower during the daytime in the NIV group, but not during the night. The NIV group also had an increased mean percentage of Stage 1 sleep than the INV group and a reduced percent of SWS. When one patient with non-REM sleep that was inconsistent with normal due to the absence of typical Stage N2 sleep figures was excluded, ${ }^{5}$ the significance for the difference of SWS was lost $(p=0.07)$. REM sleep was virtually absent in both groups.
Sleep of NIV compared to INV patients was also more fragmented, with increased frequency of arousals and awakenings (Table 2). Although arousals as a result of ventilator asynchrony tended to be more frequent in NIV patients, the difference was not statistically significant. Only two NIV patients had respiratory event related arousals (7.4 and 8.4/hour) during spontaneous breathing and none during NIV use. Therefore, median number of arousals related to respiratory 
events was negligible ( 0 in both groups; $p>0.05$ ). Sleep architecture and circadian rhythm varied greatly between patients (Figure 1).

\section{Vital Signs and Duration of Noninvasive Ventilation Use}

Vital signs (including heart rate, respiratory rate, diastolic blood pressure, and oxygen saturation) recorded hourly during the study were similar in both groups except for systolic blood pressure, which was lower in the NIV group than the INV group (median systolic blood pressure of 107 [100-112] versus 123 [112-125]; $p=0.04)$.

NIV was applied continuously in two patients and intermittently in six patients. For those six patients, NIV was applied $70 \%$ of the total recording time with a mean sleep efficiency of $39 \%$, and it was removed $30 \%$ of the time (with application of $\mathrm{O}_{2}$ via nasal cannulae) with a mean sleep efficiency of $7.8 \%(p=0.045)$.

\section{Use of Sedation and Analgesia}

NIV patients received more intermittent sedation and less sedation overall than INV patients $(p=0.001)$. Two of the NIV patients received no sedation; the other NIV patients received morphine (two patients), diphenhydramine, (two), lorazepam (one), quetiapine (one), and hydromorphone (one), all intermittently. The sedative agents used by the INV group were fentanyl (seven patients), propofol (three), midazolam (five), hydromorphone (two), quetiapine (one), and lorazepam (one). Two INV patients received only continuous sedation protocols, whereas six of them received not only continuous sedation, but also intermittent boluses.

\section{Long-Term Outcomes}

Three of the NIV patients were intubated and eventually died (1-5 days and 4-11 days after the termination of the study, respectively), and two of the INV patients died (10 days after the termination of the study). The mean TST of the NIV patients requiring intubation was longer than other NIV patients, but this was not statistically significant (9.2 \pm 4.0 [1.2-13.8] versus 6.2 \pm 1.8 [0.610.7] hours, respectively; $p>0.05$ ), whereas the mean APACHE II score was higher on the day of the study $(26.0 \pm 2.0$ [22-28] versus $17.3 \pm 1.8$ [1322], respectively; $p=0.02$ ). Mean hospital and ICU lengths of stay were similar between the NIV and INV groups (18 \pm 1.1 [10-23] versus 19.8 \pm 3.1 [7-35] days, and 14.0 $\pm 2.2[5-21]$ versus $14.9 \pm 3.2[5-34]$ days, respectively; $p>0.05$ ).

\section{DISCUSSION}

This first pilot study comparing sleep architecture in patients receiving NIV and INV for management of ARF in the ICU found that NIV patients 1) were sicker based on higher APACHE II scores and lower $\mathrm{pH} ; 2$ ) slept less and lighter in 24 hour; 3) had more fragmented sleep; and 4) received less sedation. Fragmentation of sleep was mainly due to spontaneous arousals and awakenings. Although arousals due to ventilator asynchronies (including asynchronies due to air leakages) were more common during NIV than INV use the difference was not significant. Noise and patient care contributed little to the sleep fragmentation. Lengths of stay and mortality outcomes were similar between groups although the number of patients was small.

Sleep abnormalities in critically ill patients have been repeatedly reported over the past three

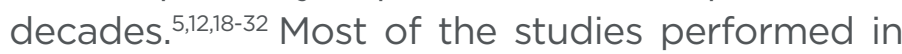
intubated patients reported normal or near normal duration of sleep (6-8 hours/24 hours), ${ }^{18-20,28,32}$ although some studies revealed a decreased TST, 20,22,23,31 and others showed longer durations of sleep..$^{21,24,30}$ In this study, patients on NIV had shorter TST with lower sleep efficiency than patients receiving INV. The TST of NIV patients from this study were similar to those reported for NIV patients previously, 5,12 whereas INV patients would have been considered 'long sleepers' as described by others. ${ }^{21,24,30}$ The shorter TST of NIV compared to INV patients could be related to many factors including the higher severity of illness of this study's NIV population and more frequent and continuous use of sedatives in the INV patients. Furthermore, the NIV patients had received less ventilatory assistance than the INV patients (median $1^{\text {st }}$ day versus $4^{\text {th }}$ day) before enrolment and could have been less well acclimated. Furthermore, patients on both types of ventilation varied greatly in sleep duration, ranging from 0.6 to 15.2 hours over 24 hours. This corresponds with prior studies ${ }^{18,19,32}$ and probably reflects the great variability between patients in acuity of illness, use of and responsiveness to medications, and many other possible factors. ${ }^{7}$ 
A

Sleep stage
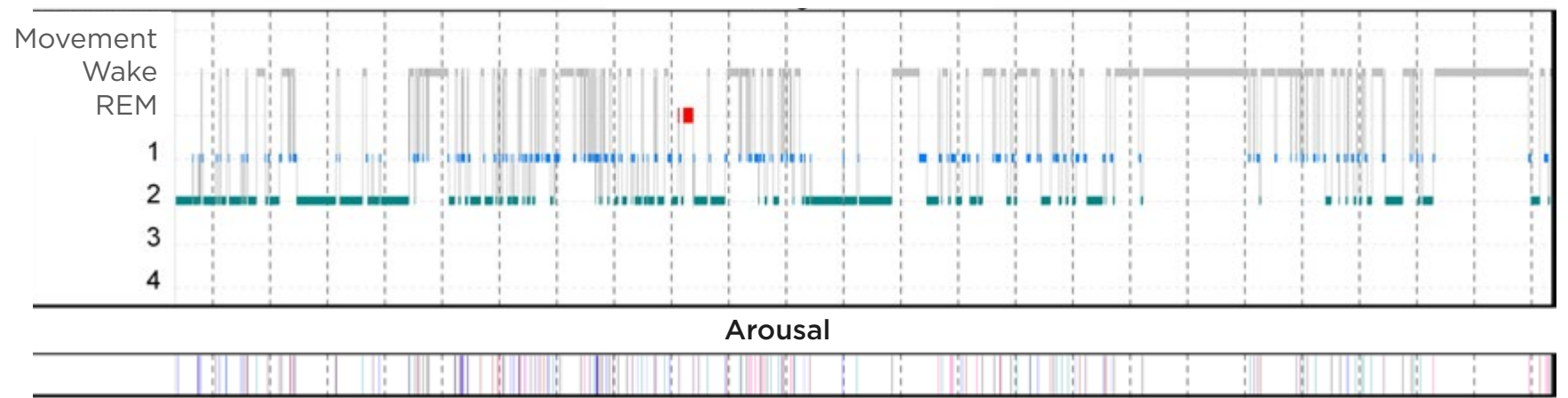

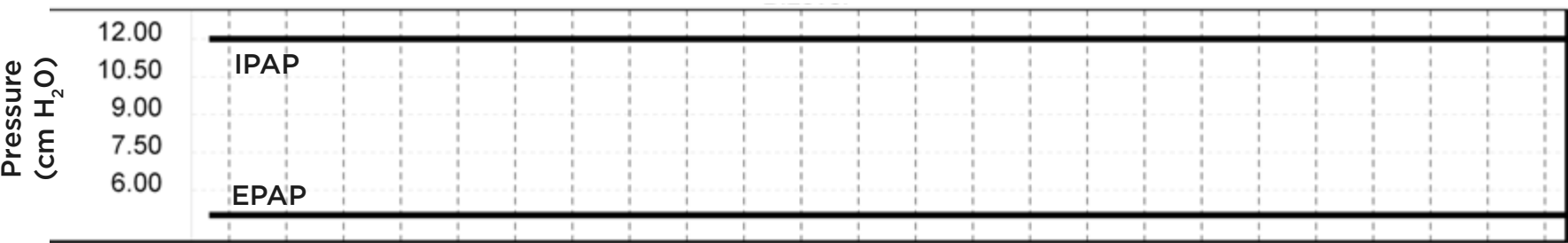

B

Sleep stage
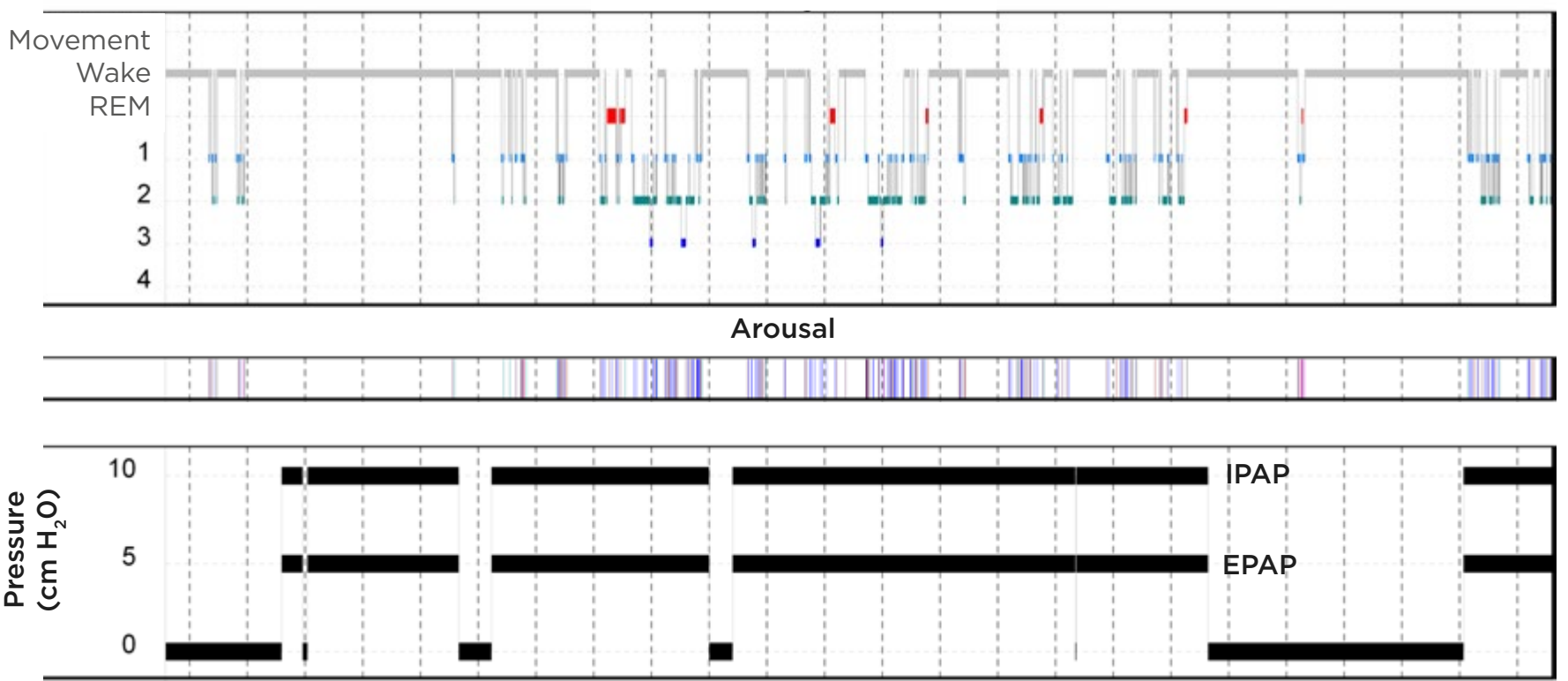

C

Sleep stage

Movement

Wake

REM

\begin{tabular}{l|l|l|}
1 & & \\
2 & & \\
3 & & \\
4 & &
\end{tabular}

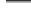

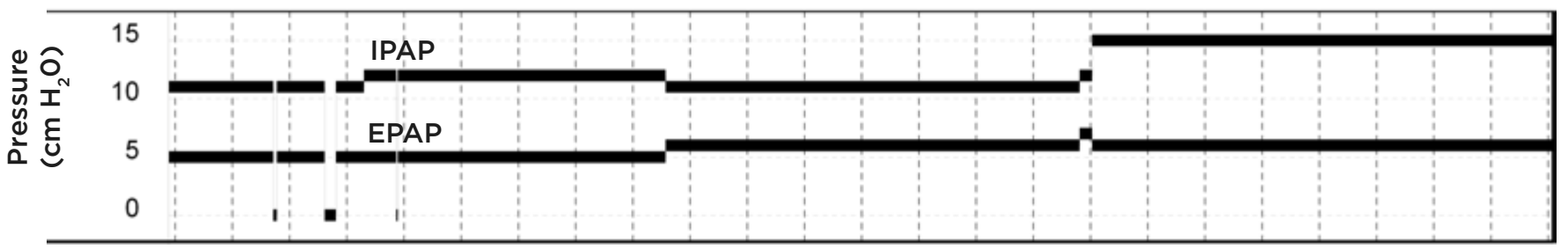


D

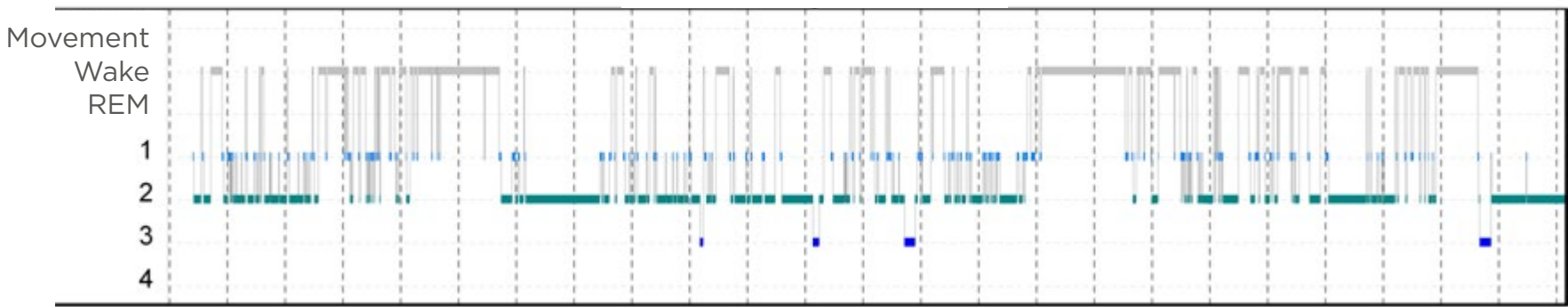

Arousal

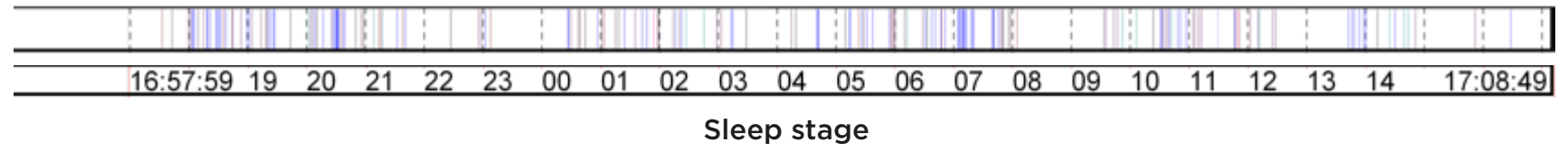

E

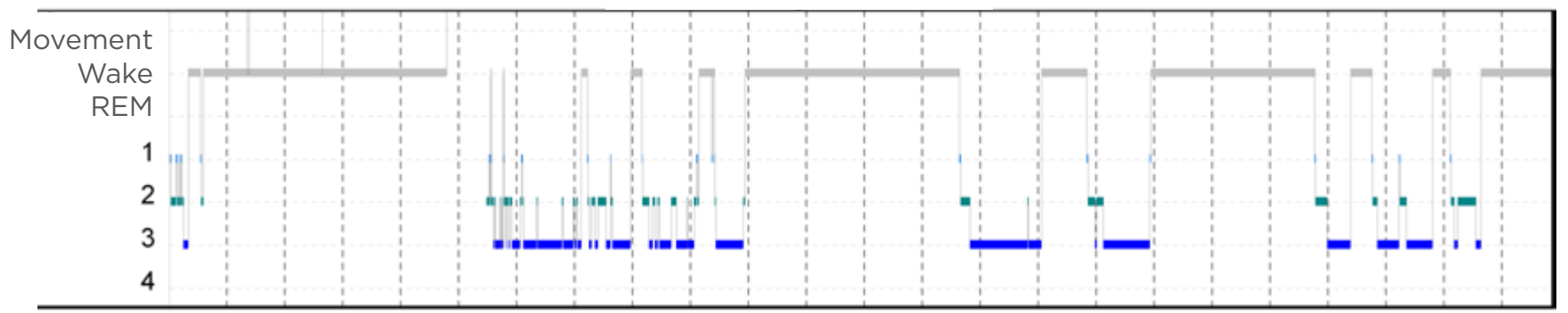

Arousal

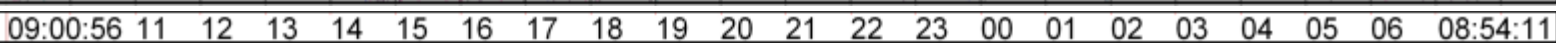

F

Sleep stage

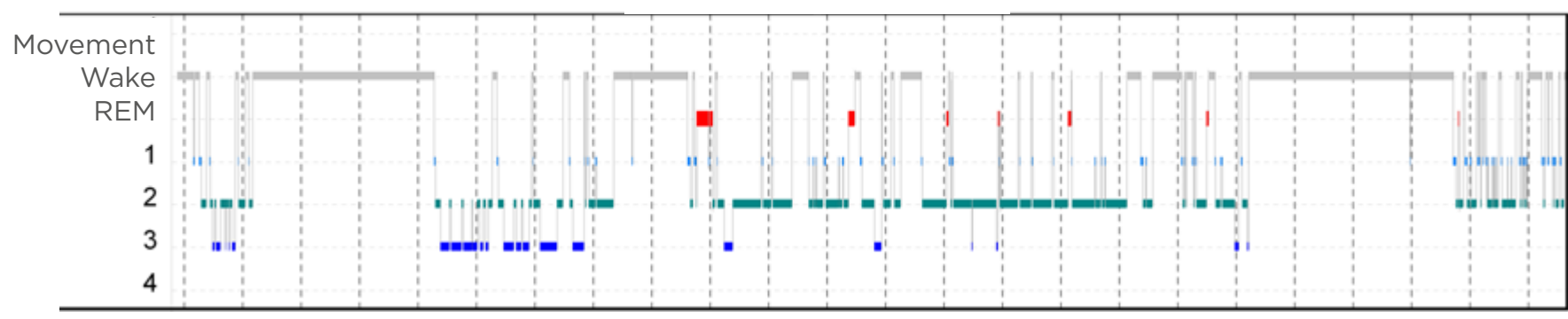

Arousal

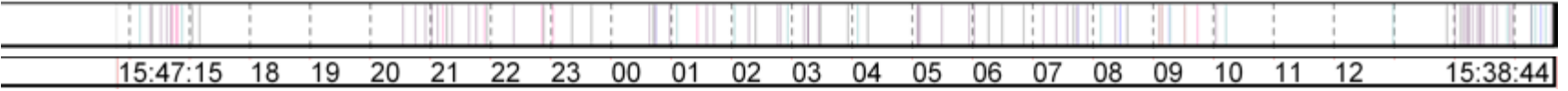

Figure 1: 24-hour hypnograms of six patients.

A, B, C in noninvasive ventilation group and $\mathbf{D}, \mathbf{E}, \mathbf{F}$ in invasive mechanical ventilation group ( $X$ axis is the timeline). The circadian rhythm was partly conserved in some patients ( $\mathbf{B}$ and $\mathbf{F}$ ) but lost in others ( $\mathbf{A}$ and $\mathbf{D}$ ). Total sleep time was severely decreased in two of the noninvasive ventilation patients who slept $<2$ hours (C). One of the patients on invasive mechanical ventilation had an abnormal sleep pattern with increased slow-wave sleep (E).

EPAP: expiratory positive airway pressure; IPAP: inspiratory positive airway pressure; REM: rapid eye movement.

Previous ICU studies have shown disruption of the circadian pattern with increased napping so that daytime sleep reached up to an average of $60 \%$ of TST in a 24 -hour cycle. ${ }^{18-20}$ Similarly, the patients of this study in both groups slept around $60 \%$ of the TST during the daytime.

Patients on mechanical ventilation in ICU generally have diminished sleep quality, characterised by an increase in the proportion of time spent in 'light' stages of sleep and a decrease in the proportion or even absence of slow wave or REM sleep. 19,23,24,31,32 Consistent with these observations, patients of this study, especially the ones on NIV, also manifested a greater than normal proportion of time spent in lighter stages of sleep. REM sleep was observed only in four NIV and two INV patients with a range 
of 1.2 to $11.5 \%$ of TST. Still, sleep efficiency was better preserved during application of NIV compared to breaks off NIV, consistent with prior observations, but this might be related to increased patient activities during breaks. ${ }^{12}$

Two prior PSG studies have evaluated sleep in hypercapnic patients receiving NIV in an ICU for 17 hours. ${ }^{5,12}$ Median TST was 5.8 hours with $68-74 \%$ of sleep occurring during at night. REM sleep deprivation (7-11\% of TST) and severe sleep fragmentation (24-33/hour) were noted. Based on the assumption that patients were awake between 8 AM and 3 PM (because of nursing activities and diagnostic testing), the authors surmised that circadian rhythm and the proportion of SWS (22\%) were better preserved with NIV compared to earlier studies on patients receiving $\mid \mathrm{NV}^{18-20}$ The latter findings conflict with findings from this study. Here, sleep was directly compared during NIV and INV in contemporaneous patients in the same ICU rather than earlier studies and monitored for the entire 24 hours rather than 17 hours. Furthermore, patients had greater acuity of illness, more acidaemia ( $\mathrm{pH}: 7.25$ versus 7.32), an earlier period of observation during ventilator assistance and greater use of sedative/analgesic medications, which may account for some of the differences.

The study authors encountered more sleep fragmentation, attributable mainly to an increased number of arousals and awakenings, in NIV group compared to INV group. Common contributors to disrupted sleep include factors associated with the ICU environment, critical illness, medications, and mechanical ventilation. ${ }^{1,3,8}$ The higher level of sleep disruptions with more frequent spontaneous arousals and awakenings during NIV compared to INV could have been related to the higher severity of critical illness in NIV patients, less frequent use of continuous sedation, or other unmeasured factors. On the other hand, it should be mentioned that the arousal index for both groups still seems to be within normal limits ${ }^{33}$ and the sleep fragmentation index for the NIV group to be less than the prior studies (22/hour versus 33/hour), 5,12 which could be attributed to differences in severity of the disease, ventilator, or sedative management as mentioned above.

Patients on mechanical ventilation usually receive intermittent or continuous sedation to control agitation and to improve patient-ventilator interactions. Even though benzodiazepines and propofol at night can increase TST or decrease sleep fragmentation, ${ }^{21,34,35}$ they can lead to significant disturbances of sleep architecture, with suppression of SWS and REM sleep. 4,7,36 While two of the NIV patients were not receiving any sedatives or analgesics, the rest were receiving less sedation on a more intermittent basis than INV patients. This difference could have contributed to the decreased quantity and quality of sleep in the NIV compared to the INV patients.

The presented study has a number of strengths including the complete and continuously observed PSG recordings for 24 hours, the prospective design, and the careful analysis including recording of noise and disruptions by caregivers. It also has important limitations including the small sample size that precludes the ability to draw inferences about the effects of disrupted sleep on clinical outcomes such as lengths of stay or mortality. Furthermore, patients were not randomised; therefore, selection bias was likely to create inevitable differences between the NIV and INV groups. Additionally, the authors were unable to control confounding variables such as use of medications and sedation level, severity of illness, fluctuations in patient illness, presence of undiagnosed sleep apnoea, activities of medical personnel, and clinical testing. On the other hand, the study was performed as a pilot to attract attention to this topic. The study was labour intensive, difficult to enrol (only $5 \%$ of screened patients), and technically challenging to administer in an ICU given interference with delivery of clinical care was prohibited. Furthermore, use of the Rechtsschaffen and Kales ${ }^{16}$ sleep scoring manual has not been well validated in critically ill patients and is confounded by poor inter-observer reliability, except for REM sleep. ${ }^{37}$

\section{CONCLUSION}

The study demonstrated that compared to INV, critically ill patients using NIV for ARF had lower quantity and quality of sleep. These data are the first to suggest that sleep time and quality are compromised to a greater extent with NIV than INV. On the other hand, keeping the patient on NIV can protect against other adverse events 


\section{References}

1. Rittayamai $\mathrm{N}$ et al. Positive and negative effects of mechanical ventilation on sleep in the ICU: a review with clinical recommendations. Intensive Care Med. 2016;42(4):531-41.

2. Pisani MA et al. Sleep in the intensive care unit. Am J Respir Crit Care Med. 2015;191(7):731-8.

3. Ozsancak A et al. Sleep and mechanical ventilation. Crit Care Clin. 2008;24(3):517-31.

4. Figueroa-Ramos $\mathrm{Ml}$ et al. Sleep and delirium in ICU patients: a review of mechanisms and manifestations. Intensive Care Med. 2009;35(5):781-95.

5. Roche Campo F et al. Poor sleep quality is associated with late noninvasive ventilation failure in patients with acute hypercapnic respiratory failure. Crit Care Med. 2010;38(2):477-85.

6. Chen HI, Tang YR. Sleep loss impairs inspiratory muscle endurance. Am Rev Respir Dis. 1989;140(4):907-9.

7. Parthasarathy S. Sleep during mechanical ventilation. Curr Opin Pulm Med. 2004;10(6):489-94.

8. Hardin KA. Sleep in the ICU: potential mechanisms and clinical implications. Chest. 2009;136(1):284-94.

9. Walkey AJ, Wiener RS. Use of noninvasive ventilation in patients with acute respiratory failure, 20002009: a population-based study. Ann Am Thorac Soc. 2013;10(1):10-7.

10. Ozsancak Ugurlu A et al. Use and outcomes of noninvasive positive pressure ventilation in acute care hospitals in Massachusetts. Chest. 2014;145(5):964-71.

11. Demoule $A$ et al. Changing use of noninvasive ventilation in critically ill patients: trends over 15 years in francophone countries. Intensive Care Med. 2016;42(1):82-92.

12. Cordoba-Izquierdo A et al. Sleep in hypercapnic critical care patients under noninvasive ventilation: conventional versus dedicated ventilators. Crit Care Med. 2012;41(1):60-8.

13. Teschler $\mathrm{H}$ et al. Effect of mouth leak on effectiveness of nasal bilevel ventilatory assistance and sleep architecture. Eur Respir J. 1999;14:1251-7.
14. Meyer TJ et al. Air leaking through the mouth during nocturnal nasal ventilation: effect on sleep quality. Sleep. 1997;20:561-9.

15. Tufts Medical Center. Effects of invasive and noninvasive mechanical ventilation on sleep in the intensive care unit (ICU). NCTO0638339. https://clinicaltrials.gov/ct2/show/ NCT00638339.

16. Silber $\mathrm{MH}$ et al. The visual scoring of sleep in adults. J Clin Sleep Med. 2007;3: 121-31.

17. Iber $C$ et al., The AASM manual for the scoring of sleep and associated events: rules, terminology and technical specifications (2207) 1st edition, Darien: American Academy of Sleep Medicine.

18. Cooper AB et al. Sleep in critically ill patients requiring mechanical ventilation. Chest. 2000;117(3):809-18.

19. Freedman NS et al. Abnormal sleep/wake cycles and the effect of environmental noise on sleep disruption in the intensive care unit. Am J Respir Crit Care Med. 2001;163(2):451-7.

20. Gabor JY et al. Contribution of the intensive care unit environment to sleep disruption in mechanically ventilated patients and healthy subjects. Am J Respir Crit Care Med. 2003 167(5):708-15.

21. Hardin KA et al. Sleep in critically ill chemically paralyzed patients requiring mechanical ventilation. Chest. 2006;129(6):1468-77.

22. Hilton BA. Quantity and quality of patients' sleep and sleep-disturbing factors in a respiratory intensive care unit. J Adv Nurs. 1976;1(6):453-68.

23. Aurell J, Elmqvist D. Sleep in the surgical intensive care unit: continuous polygraphic recording of sleep in nine patients receiving postoperative care. Br Med J (Clin Res Ed). 1985;290(6474):1029-32.

24. Gottschlich MM et al. The 1994 clinical research award. A prospective clinical study of the polysomnographic stages of sleep after burn injury. $J$ Burn Care Rehabil. 1994;15(6):486-92.

25. Parthasarathy S, Tobin MJ. Effect of ventilator mode on sleep quality in critically ill patients. Am J Respir Crit Care Med. 2002;166(11):1423-9.

26. Toublanc B et al. Assist-control ventilation vs. low levels of pressure support ventilation on sleep quality in intubated ICU patients. Intensive Care Med. 2007;33(7):1148-54.

27. Bosma K et al. Patient-ventilator interaction and sleep in mechanically ventilated patients: pressure support versus proportional assist ventilation. Crit Care Med. 2007;35(4):1048-54.

28. Cabello B et al. Sleep quality in mechanically ventilated patients: comparison of three ventilatory modes. Crit Care Med. 2008;36(6):1749-55.

29. Fanfulla $F$ et al. Effects of different ventilator settings on sleep and inspiratory effort in patients with neuromuscular disease. Am J Respir Crit Care Med. 2005; 172(5):619-24.

30. Fanfulla $\mathrm{F}$ et al. Sleep disturbances in patients admitted to a step-down unit after ICU discharge: the role of mechanical ventilation. Sleep. 2011;34(3):355-62.

31. Beecroft JM et al. Sleep monitoring in the intensive care unit: comparison of nurse assessment, actigraphy and polysomnography. Intensive Care Med. 2008;34(11):2076-83.

32. Friese RS et al. Quantity and quality of sleep in the surgical intensive care unit: are our patients sleeping? J Trauma. 2007;63(6):1210-4

33. Bonnet $\mathrm{MH}$, Arand DL. EEG arousal norms by age. J Clin Sleep Med. 2007;3(6): 271-4

34. Treggiari-Venzi M et al. Overnight sedation with midazolam or propofol in the ICU: effects on sleep quality, anxiety and depression. Intensive Care Med. 1996;22:1186-90.

35. Alexopoulou $\mathrm{C}$ et al. Sleep during proportional-assist ventilation with load-adjustable gain factors in critically ill patients. Intensive Care Med. 2007;33(7):1139-47.

36. Kondili E et al. Effects of propofol on sleep quality in mechanically ventilated critically ill patients: a physiological study. Intensive Care Med. 2012;38:1640-6.

37. Ambrogio $\mathrm{C}$ et al. Assessment of sleep in ventilator-supported critically III patients. Sleep. 2008;31(11):1559-68. 